\title{
LAS DOS CLASES DE ACTIVIDAD MUSCULAR PROVOCADA: LA NEUROMUSCULAR Y LA IDIOMUSCULAR. SINOPSIS FISIOPATOLOGICA Y CLINICA
}

\author{
LUIS Barraquer-Bordas
}

Cuando, el 4 de junio de 1922, presentó Luís Barraquer-Roviralta su memoria sobre el valor semiológico de la contracción idiomuscular ${ }^{1}$; señaló de una manera clara y taxativa la distinción que debe hacerse entre las respuestas musculares mediatizadas por el sistema nervioso y las idiomusculares, situando esta distinción, con todo su valor y su significación semiológica, en el campo de la clínica. Nosotros procuraremos en el presente trabajo ahondar en esta distinción, puntualizando su significación fisiopatológica y clínica. Pues es verdad que, aunque conocida por los clá. sicos y admitida por todos, no suele ser interpretada en sus justos términos.

Empezaremos precisando en que sentido emplearemos aquí la expresión actividad muscular provocada. Por actividad muscular entenderemos sim. plemente la actuación funcional del músculo, su contracción, se acompañe o no de trabajo en el sentido físico. Y nos referiremos solamente a la musculatura esquelética. Por otra parte, convendremos en considerar como "provocada" - para los fines de este trabajo - la actividad muscular desencadenada por estímulos procedentes del exterior del organismo (medio ambiente) y regulados por nosotros mismos. Concretamente nos referiremos como estímulos de tal provocación a las maniobras de percusión clí. nica realizadas habitualmente con el martillo llamado de reflejos. La percusión provocadora podrá recaer sobre el propio músculo, en puntos vecinos a éste o aun en puntos lejanos. Podemos decir, pues, que entenderemos aquí por actividad muscular provocada, la contracción del músculo esquelético subsiguiente a la percusión mediante el martillo de reflejos. A esta actividad muscular provocada le llamaremos, así en conjunto, en general, respuesta.

En condiciones normales el músculo esquelético está unido al sistema nervioso central por una doble conexión, aferente y eferente, que, según demostró Sherrington ${ }^{2}$, corresponde a los mismos segmentos medulares. La conexión aferente toma su origen en los receptores ánulo-espirales y en "flower spray", que son específicamente sensibles a la tracción - pasiva por estar situados "en derivación" respecto a los elementos contráctiles (Fulton y Pi-Suñer ${ }^{3}$ ). La conexión eferente termina en la llamada placa motriz, que es la estructura a través de la cual se vierten al músculo los es-

Trabajo de la Clínica de Neurología del Hospital de la Sta. Cruz y S. Pablo (Director: Prof. L. Barraquer-Ferré), Barcelona, España. 
tímulos vehiculados por la vía final común. Pero aunque este es el cauce específico para que la irritabilidad y la contractibilidad del músculo sean solicitadas, no es el único. Y entre los otros cauces posibles está la acción mecánica del mundo exterior, la percusión con el martillo, p. ej.

Ahora bien, aun cuando el músculo sea solicitado a la contracción sin utilizar su nervio motor y la placa motriz, no por ello la respuesta asi obtenida está absolutamente desligada c independicnte del control del sistema nervioso, sino que las características de tal respuesta dependen precisamente en todo momento del grado de autonomía que este sistema concede al tejido muscular.

Después de esta introducción podemos ya decir que existen dos clases de actividad muscular provocada o respuestas del músculo esquelético: a) Si la percusión determina un alargamiento suficientemente súbito e intenso del músculo, estimula al órgano terminal sensible a la tracción y aparece una contracción del mismo músculo desencadenada a través del sistema nervioso. El sistema alıatómico y funcional músculo-sistema nervioso-músculo proporciona, pues, al ser estimulado de este modo, uni respuesta que llamaremos neuromuscular. Es la respuesta refleja o reflejo en el sentido clásico de los reflejos musculares propioceptivos e miotáticos. b) $\mathrm{Si}$ la percusión despierta directamente la irritabilidad propia del músculo, pone en marcha tambien directamente su contractibilidad y se obtiene así una respuesta autóctona, una contracción del músculo de crigen local. Esta actividad muscular directamente provocada, no mediatizada, aunque controlada indirectamente por el sistema nervioso, es la respuesia o contracción idiomuscular.

Para obtener la respuesta neuromuscular lo esencial es producir una dístensión del músculo suficientemente intensa y súbita - de acuerdo con el estado de "irritabilidad profunda" - aplíquese donde se aplique la percusión, mientras que, para producir la respuesta idiomuscular es necesario percutir directamente sobre el propio músculo.

Las respuestas reflejas propioceptivas neuromusculares tienen su base, de acuerdo con los factores que antes hemos señalado, en el "stretch reflex" o reflejo de tracción. Podemos definir éste como la respuesta refleja, la contracción, que nos suministra un músculo cuando éste propio músculo es estimulado mediante la elongación o distensión de sus fibras. Creed y col. ${ }^{4}$ lo valoran como la resistencia ofrecida por un músculo en plena conexión con el sistema nervioso. El reflejo de tracción es, pues, podemos decir tambien, la resistencia refleja que el músculo opone a ser distendido.

En clínica neurológica se utilizan cotidianamente unas respuestas neuromusculares cuya esencia descansa en el reflejo de tracción. Son los llamados por los autores clásicos, reflejos tendinosos ("tendon reflex" de los anglosajones, "Sehnenreflexe" de los alemanes, "réflexes téndineux" de los franceses) y en otros casos, según el punto de su provocación clínica, reflejos óseos, periósticos, fasciales, aponeuróticos, etc. Muchas veces estas respuestas reflejas son además calificadas, en virtud de ciertas circunstancias de su provocación, de paradójicas, invertidas, antagonistas. Algunos 
autores llaman, más inespecifica y menos comprometidamente, a estas respuestas reflejas, reflejos profundos ("deep reflex" de los anglosajones, "Tiefenreflexe" de los autores alemanes). Otros, siguiendo a Gowers 5 , quién rechazó por primera vez la nomenclatura clásica, los denominan reflejos miotáticos ("myotatic reflex"), haciendo referencia a que se producen por maniobras de distensión muscular. Todavía otros autores, apurando e insistiendo en su base fisiológica, los califican de reflejos propios de los mús. culos ("Eigenreflexe menschlicher Muskeln" ${ }^{6}$ ) o "muscle stretch reflex" ". Se trata de respuestas reflejas fásicas por tracción muscular provocadas clínicamente por percusión y es nuestra intención analizar brevemente sus relaciones con el reflejo de tracción - en el sentido fisiológico - como punto de partida para su adecuada compreensión.

Todas estas respuestas son, en realidad, modalidades fásicas particulares del reflejo de tracción provocadas a voluntad mediante percusión en puntos electivos. No pueden por ello ser llamadas genéricamente "strech reflexes" porque son solo — como venimos diciendo - uma modalidad particular de este reflejo, una modalidad que es puesta en marcha por nosotros valiéendonos de ciertas circunstancias de provocación propias del método de exámen clínico y que representa para el organismo, concretamente para el sistema neuromuscular puesto en función, una eventualidad desacostumbrada y ocasional. El concepto de estas respuestas reflejas no puede ser ni exclusivamente fundamentado en la fisiologia ni exclusivamente derivado del método de exámen. Se trata de un concepto bipolar que se debe a la vez a ambas cuestiones. Tal respuesta refleja y su valoración clínica dependen realmente de una relación, de la relación entre el estado de irritabilidad profunda del músculo que proporciona la respuesta por tracción - con todas las características individuales de esta respuesta en cada caso concreto - y el punto en el cual ha sido aplicada la percusión provocadora - con las caracteristicas de intensidad, dirección, brusquedad, de esta misma percusión. Es así como la fisiología o fisiopatología y el método clínico vienen a darse la mano para establecer una relación que proporciona a la respuesta refleja producida - cuando esta relación es justamente conocida y apreciada - todo su valor semiológico. De este modo la semiología viene a ser la interpretación de la fisiopatología en función del método de exámen clínico. Y el reflejo clínico al que nos venimos refiriendo, la respuesta refleja fásica por tracción de un músculo determinado en función del punto de aplicación de la percusión provocadora y de las características de intensidad, dirección, brusquedad, etc., de esta percusión.

En una comunicación presentada a la Reunión Inaugural de la Sociedad Española de Neurología (Barcelona, 19 de diciembre de 1949) analizamos detalladamente los diferentes elementos que entran en la definición conceptual de las respuestas reflejas a las que nos venimos refiriendo, tal como se presentan en la clínica ${ }^{8}$. Su nota esencial es el ser manifestaciones del reflejo de tracción, pero lo que las individualiza y matiza son las circuns. tancias de su provocación clínica. Junto a ello tienen una serie de cuali- 
dades o atributos, muy importantes, pero que no son su rasgo esencial. Entre ellos está el ser "antagonistas", en el sentido con que André-Thomas e Ajuriaguerra ${ }^{9}$ utilizan esta palabra. Pero este rasgo, importante, no puede utilizarse por sí solo para definir su naturaleza, como pretende este autor.

Wartenberg, en su libro ${ }^{7}$ ya clásico (1945), ha demostrado rotundamente la falsedad de las concepciones clásicas y la falacia de la nomenclatura habitual. Su trabajo de sistematización, de unificación y de síntesis debe ser cuidadosamente atendido y ha aportado un considerable beneficio a la neurología científica. Pero la necesidad de valorar en la clínica las circunstancias de la provocación obliga a menudo a adoptar una visión más amplia que la estrictamente fisiológica mantenida por este autor. La respuesta refleja fásica de tracción clínicamente provocada no es simplemente el reflejo de tracción de un músculo determiriado, sino que es una modalidad particular de este reflejo producida bajo determinadas circunstancias propias del método de exploración clínica. Monrad-Krohn ${ }^{10}$, en la caballerosa polémica sostenida con Wartenberg, hace ver la necesiảad de atender al punto de provocación para definir globalmente el reflejo en la práctica clínica, pero él mismo cuando desarrolla este capítulo ${ }^{11}$ no logra tampoco seguir una pauta sistemática.

Nosotros no proponemos una nueva nomenclatura determinada. Solo suscribimos con Wartenberg la condenación de la concepción clásica y exponemos sumariamente las razones a que una concepción y una nomenclatura justas deberán forzosamente atender.

No es necesario aquí insistir en la crítica de aquellos que han utilizado las expresiones de "reflejo tendinoso" y similares, sustentando un criterio fisiológico totalmente erróneo. Pero queremos referirnos a ciertas opiniones que por la forma en que fueron expuestas y por el prestigio de los autores que las emitieron, tienen una especial significación para juzgar la desorientación que sobre cuestiones tan fundamentales ha reinado habitualmente. Así en efecto, Dejerine y Sicard, independienternente, pretendieron sistematizar las relaciones entre respuesta refleja y contracción idiomuscular, aceptando la existencia de tres procesos distintos. Para Dejerine ${ }^{12}$, estos procesos serían: reflejo tendinoso, reflejo neuromuscular y contracción idiomuscular. Para Sicard y Cantaloube ${ }^{13}$ : reflejo tendinoso, reflejo muscular y reacción idiomuscular. Nosotros hicimos en el trabajo antes citado $^{8}$ una crítica detallada de estas falsas concepciones. Por ello las juz. garemos aquí brevemente. Por una parte, la noción de reflejo tendinoso que mantienen estos autores es puramente empírica y se basa únicamente en el punto de su provocación. El concepto de "reflejo neuromuscular" en el sentido de Dejerine y de "reflejo muscular" en el sentido de Sicard y Cantaloube es un concepto equívoco, que se funda exclusivamente en su provocación por percusión del propio músculo y en su irradiación hasta el tendón. En realidad, puede tratarse tanto de un verdadero reflejo como de una respuesta idiomuscular. Y, finalmente, el criterio que estos autores utilizan para identificar a esta última depende exelusivamente de las apariencias y de una limitación prejuzgada y errónea de sus condiciones 
de producción. Estas condiciones serán analizadas más adelante en sus términos de realidad.

Por otra parte, una muestra escogida de la superficialidad e incorreción con que los clínicos suelen juzgar la naturaleza de las respuestas reflejas de que hemos venido tratando, la tenemos en el prólogo de una reciente obra dedicada a un capítulo de la semiología. En el libro de AndréThomas y Ajuriaguerra ${ }^{9}$ - cuyo contenido semiológico es cuantioso, paciente, minucioso - se transcribe, en efecto, un intento de sistematización que de estas respuestas sugirió Sherrington en 1900 y que, posteriormente, fué completamente superado, en gran parte por la obra del propio neurofisiólogo inglés. André-Thomas cita este intento - que hoy no tiene sino un interés histórico - exactamente como lo hizo su maestro Dejerine ${ }^{12}$, y su devolución por él, si bien nos permite comprender por donde esta cita ha llegado, no la justifica en modo alguno. Es estrictamente necesario que los tratadistas se esfuercen de hoy en adelante en exponer en su verdadero punto esta cuestión y dar de los reflejos a que nos referimos una debida interpretación conceptual.

Para que esta interpretación sea completa deberá partirse de su naturaleza fisiológica, pero no deberán descuidarse sus cualidades más sobresalientes (antagonista, etc.) ni las circunstancias más destacadas de su provocación. Es así como apreciaremos toda la semejanza y toda la diferencia que existe entre el reflejo de tracción producido experimentalmente en el cuadríceps de una rana descerebrada y el reflejo clínico de este mismo músculo en el hombre. Acentuando las distinciones diremos que la difusión del estímulo hasta otros músculos muchas veces, la modificación de las características de la respuesta por el reflejo de los músculos antagonistas al ser distendidos y aún el mismo modo de comportarse el indivíduo durante el exámen clínico, son notas que prestan a la clínica humana - a la clínica personal - su sello definidor peculiar.

Finalmente, queremos hacer referencia a una expresión habituálmente empleada por los clínicos para referirse a ciertas circunstancias de la producción de estos reflejos. Trátase de la expresión zona reflexógena y es. pecialmente del llamado aumento, ampliación o extensión de la zona reflexógena. La única zona verdaderamente reflexógena de los reflejos a que nos referimos es el receptor o aferente de tracción. La zona de provocación clínica, la de aplicación de la percusión, no merece en modo alguno este título, ya que no tiene ilinguna participación activa en el origen del reflejo. Por esto Wartenberg ${ }^{7}$ ha podido decir que la expresión de "an extension of the reflexogenous zones" es un término "incorrect and misleading". Ahora bien, como que el fenómeno en sí - llámese como se deba llamar - ocurre en la clínica y tiene una significación semiológica considerable, es menester darle una denominación. Nosotros hemos propuesto ya en otros sitios ${ }^{8,14}$ emplear un término que haga referencia solamente a la maniobra mediante la cual se provoca el reflejo, a la circunstancia del método de exámen. Por esto, proponemos que se hable de "zona de provocación" y de "aumento, ampliación o extensión de la zona de provoca. 
ción". La expresión "extensión de la zona reflexógena" puede ser, en cambio, justamente empleada en referencia a ciertos reflejos nociceptivos ("skinmuscle reflex" de los anglosajones, "Fremdreflexe" de algunos autores alemanes).

Para completar el conocimiento de la actividad muscular que hemos llamado provocada, debemos referirnos ahora a una clase de contracción muscular liabitualmente poco estudiada, la respuesta idiomuscular. Según Barraquer-Roviralta ${ }^{1}$ la contracción idiomuscular se compone de dos fases: depresión y propulsión muscular. Inmediatamente después de la excitación - p. ej. por percusión - el músculo se deprime longitidinalmente en una extensión transversal correspondiente al diámetro del cuerpo excitador, depresión que no puede explicarse solo por una acción mecánica primaria. Casi simultáneamente empieza la formación del nudo en el misino punto deprimido y solo desaparece lentamente. Limitación y lentitud son dos características fundamentales de la respuesta idiomuscular que sirven para diferenciarla de la neuromuscular. La limitación, sin embargo, es solamente en anchura, ya que en longitud la contracción idiomuscular puede extenderse hasta el tendón y, cuando está aumentada, puede determinar por esta vía un desplazamiento de la articulación que el músculo moviliza. El no aceptar este hecho fué precisamente uno de los errores básicos de la concepción de Sicard y Cantaloube ${ }^{13}$.

El origen de la respuesta idiomuscular estriba en la irritabilidad y contractibilidad de las fibras musculares estriadas, propiedades que en tal caso son solicitadas directamente, sin utilizar el camino específico que es la placa motriz.

En la respuesta idiomuscular cada fibra responde funcionalmente según el grado de excitación, y ésta es proporcional a la distensión pasiva determinada por el agente provocador. Como ha sugerido Torruella-Font ${ }^{15}$, se trata de una aplicación particular de la ley de Starling. Dado que la superficie del excitador suele ser piramidal o semiesférica, la graduación de la intensidad de la percusión es igualmente piramidal o semiesférica.

Entre los individuos normales la contracción idiomuscular of rece diferencias bastante considerables. En general, puede enunciarse el principio de que es tanto más evidente cuanto menor es el tono muscular. Ello es debido a que en tales casos es máxima la distensión pasiva provocada en las propias fibras musculares, y por tanto, máximo el estímulo.

En los estados patológicos es tambien este principio el que informa las modificaciones experimentadas por la respuesta idiomuscular; ésta es siempre proporcional a la distensión provocada en las fibras musculares y esta distensión está em relación inversa con el estado de tono.

Luego podemos anunciar que: 1) la respuesta idiomuscular estará aumentada en los estados de hipotonia; 2) la respuesta idiomuscular estará disminuída en los estados de hipertonía. $Y$ finalmente, otro principio, bien comprensible, rige también la graduación de la intensidad de esta respuesta, y es que: 3) la respuesta idiomuscular está disminuída en los casos de 
atrofía muscular y puede llegar a perderse en los estados de degeneración y esclerosis del músculo esquelético.

Para los propósitos clínicos es de gran interés conocer las relaciones entre las respuestas neuromuscular e idiomuscular en estado normal y sus modificaciones en los estados patológicos. Estas relaciones y tales modificaciones dependen del estado funcional del sistema nervioso, y su estudio puede ilustrarnos, por tanto, acerca de este estado funcional.

En condiciones normales y para un determinado músculo, suelen existir posibilidades de despertar por la maniobra clínica de percusión ambos tipos de respuesta, desde distintas zonas. Estudiar la distribución de estas zonas es estudiar el régimen habitual de convivencia clínica entre ambas respuestas. Este régimen fué descrito en forma adecuada por Luís Barraquer-Roviralta ${ }^{1}$, citando como ejemplo lo que ocurre con el músculo tríceps sural. En efecto, si nosotros percutimos sobre el tendón de Aquiles. obtendremos una contracción de este músculo por vía refleja, esto es, una respuesta neuromuscular. Contrariamente, si percutimos sobre la porción carnosa superior de esta masa muscular lograremos producir, corrientemente, una contracción de origen muscular local, es decir, una respuesta idiomuscular. Y si a distintos niveles del mismo músculo en dirección distal vamos repitiendo la percusión, llegaremos a observar que la contracción idiomuscular va disminuyendo hasta llegar a la aponeurosis tendinosa, en donde será muy tenue o nula. Si, inversamente, percutimos en dirección proximal desde el punto electivo del tendón de Aquiles hacia arriba, veremos también disminuir la respuesta neuromuscular hasta la aponeurosis de inserción donde suele quedar abolida. A esta zona en que la percusión no suele determinar respuesta ni neuromuscular (refleja) ni idiomuscular, denominó Barraquer-Roviralta" "punto neutro". Es posible que en ciertos individuos el punto neutro corresponda no a una abolición de ambas respuestas, sino a la coincidencia de las mismas, ambas por un igual de escasa intensidad.

Aunque procedimientos de detección más finos que el método clínico p. ej. la electromiografía - puedan poner de manifiesto de modo más detallado el compartamiento del músculo ante la estimulación experimental, descartando quizá la noción del punto neutro, la exposición que acabamos de hacer del régimen habitual de convivencia entre las respuestas neuromuscular e idiomuscular, tal como se presenta en la clínica, tendrá siempre un valor cierto y capital, pues descansa sobre la utilización del método básico de exámen de estas respuestas: la percusión con el martillo llamado de reflejos. Y es en este sentido en el que nosotros lo queremos valorar. Partiendo de su conocimiento nunca podrán aceptarse concepciones tan erróneas como las de Dejerine y de Sicard y Cantaloube, que antes hemos referido.

El régimen de convivencia entre las respuestas neuromuscular e idiomuscular experimenta modificaciones características en los estados de patología nerviosa, algunas de las cuales describiremos brevemente.

En los casos de espasticidad aumenta la zona de provocación de la respuesta refleja o neuromuscular, mientras que la idiomuscular se atenua o 
desaparece, de acuerdo con el grado de hipertonía. En tales casos es posible despertar la respuesta refleja no solo desde el punto habitualmente neutro, sino también de zonas más proximales, sobre la propia masa muscular.

En los estados de hipotonía la respuesta neuromuscular disminuye o desaparece, mientras que la idiomuscular se exalta, siempre y cuando la hipotonía no se acompañe de atrofía. Cuando la lesión radica en los troncos nerviosos periféricos, la hipotonía y la atrofía suelen marchar paralelas o más bien esta última sigue a aquella. Por esto, en las polineuritis y polineuropatías y en las secciones nerviosas suele exagerarse en un primer período por lo menos, la respuesta idiomuscular, pero si la atrofía avanza, disminuye y desaparece. En las afecciones que atacan primordialmente a las raíces posteriores la hipotonía es marcadísima y la atrofía es nula. En estas condiciones la respuesta refleja se pierde, al propio tiempo que la idiomuscular adquiere su exageración máxima. Esta eventualidad fué adecuadamente observada y descrita por Barraquer-Roviralta quien le atribuyó una justa significación semiológica. El pionero de la neurología clínica española designó a esta eventualidad con el nombre de "fenómeno del contraste de la contraccion "." Su descripción se refería a la tabes y especialmente a los casos de tabes baja en los que este fenómeno se aprecia claramente en el tríceps sural. En posteriores trabajos de nuestra Escuela ${ }^{16}$ se ha insistido en el valor y significación de dicho fenómeno del contraste.

En las afecciones cerebelosas la respuesta idiomuscular se exagera considerablemente, como demostró Barraquer-Roviralta ilustrándola con una iconografía muy expresiva ${ }^{1}$ que más tarde ha sido reproducida ${ }^{15 a, ~}{ }^{\text {. }}$.

En las llamadas enfermedades musculares tal respuesta experimenta modificaciones muy características.En la distrofía muscular progresiva se deprime extraordinariamente, o mejor, desaparece. Este hecho fué apreciado ya por Erb y Bechterew y a él hizo referencia Babinski ${ }^{17}$, resaltando toda su significación. Permite profundizar en la diferenciación semiológica entre la polineuritis, en la que el reflejo se pierde mientras la respuesta idiomuscular se exagera en los primeros estadios, con dicha distrofía muscular progresiva, en la cual ambas respuestas, la refleja y la idiomuscular, son comprometidas simultáneamente y paralelamente por la patología del órgano efector. Por otra parte, es característica y bien notoria la exageración de la respuesta idiomuscular en la miotonía de Thomsen y en los músculos miotónicos de la distrofía miotónica o enfermedad de Steinert.

En la práctica clínica tiene una importancia fundamental estudiar la actividad muscular provocada, las respuestas musculares, teniendo siempre presente la distinción entre las neuromusculares y las idiomusculares y refiriendo fundamentalmente cada respuesta, en primer lugar, al músculo que la proporciona, valorando luego, en segundo término, las características del método de provocación.

En otro lugar ${ }^{8}$ hemos estudiado detalladamente las respuestas neuromusculares e idiomusculares provocadas por percusión en ciertas zonas de 
las extremidades, especialmente en el plano anterior de la pierna y en el dorso del pie. En la primera de estas regiones, valoramos especialmente las maniobras de producción del reflejo del tríceps sural por "frontal-tap", de acuerdo con la síntesis de Wartenberg ${ }^{7}$. Respecto al dorso del pie insis. timos especialmente en la crítica de las sistematizaciones intentadas por Sicard y Cantaloube ${ }^{13}$ y por otros autores, exponiendo nuestras concepciones a la luz de los principios fundamentales a los cuales nos hemos venido refiriendo. Debemos remitir a este $\operatorname{trabajo}^{8}$ a los que quieran conocer con detalle esta cuestión, así como otros puntos que alí tratamos con detenimiento.

\section{SUMARIO}

El autor denomina actividad muscular provocada a la contracción del músculo esquelético subsiguiente a la percusión mediante el martillo de reflejos. Esta actividad es llamada respuesta. Existen dos clases de respuestas: la neuromuscular o refleja, proporcionada por el sistema anatómico y funcional músculo-sistema nervioso-músculo, y la idiomuscular.

El autor analiza el concepto de las respuestas reflejas fásicas por tracción muscular provocadas por el método clinico de percusión, refíriéndose a las concepciones de los clásicos, de Wartenberg, de Monrad-Krohn, etc. Expone su visión personal. Describe la respuesta idiomuscular y sus modificaciones clínicas. Estudia el régimen habitual de convivencia clínica entre la respuesta neuromuscular o refleja y la idiomuscular y las modificaciones de este régimen en los estados patológicos. Se refiere en especial ao fenómeno denominado por Barraquer-Roviralta "fenómeno del contraste de la contracción", que aparece en las lesiones de las raíces posteriores p. ej. en la tabes - y que consiste en la pérdida de la respuesta refleja con exageración de la idiomuscular.

\section{SUMMARY}

The author uses the word incited muscular activity for the contraction of the skeletal muscle following a percussion given by a reflex-testing hammer. This activity is called response. There are two classes of responses: the neuromuscular or reflex response and the idiomuscular response.

The author analyzes the concept of the phasic reflex-responses by muscular stretch incited by the clinical percussion-method, revising the conceptions of classical authors, Wartenberg, Monrad-Krohn, etc. He thereupon exposes his own ideas. The author describes the idiomuscular response and its clinical modifications. He studies the habitual regimen of clinical co-existence between the neuromuscular response and the idiomuscular response and also the modifications of this regimen in pathological conditions. He especially refers to a phenomenon called "phenomenon of contrast of the contraction" by Barraquer-Roviralta, which appears in lesions of the posterior roots - for instance, in tabes - and consists in a loss of the reflex response with increase of the idiomuscular jesponse. 


\section{BIBLIOGRAFIA}

1. Barraquer-Roviralta, L. - Valor semiológico de la contracción idiomuscular. Arch. Neurobiol., 3:2, 1922.

2. Sherrington, Ch. S. - Selected Writings, compiled and edited by Denny-Brown. Capítulo 3, pp. 94-119. Hamish Hamilton Medical Books, London, 1939.

3. Fulton, J. F. y Pi-Suñer, J. - A note concerning the probable function of various afferent end-organs in skeletal muscle. Am. J. Physiol., 83:554-562, 1928.

4. Creed, R. S., Denny-Brown, D., Eccles, J. C., Liddell, E. G. T. y Sherrington, Ch. S. - Reflex Activity of the Spinal cord. 'The Claredon Press, Oxford, 1932.

5. Gowers, W. R. - Diagnosis of Diseases of the Spinal Cord. Churchill, London, 1880, pág. 25.

6. Hoffmann, P. - Untersuchungen ïber die Eigen reflexe menschlicher Muskeln. Springer, Berlin, 1922.

7. Wartenberg, R. - The Examination of Reflexes. A simplification. The YearBook Publishers, Chicago, 1945.

8. Barraquer-Bordas, I. - Las dos clases de actividad muscular provocada: la neuromuscular y la idiomuscular. Estudio fisiopatológico y clínico. Reunión Inaugural de la Sociedad Española de Neurología. Barcelona, 19 de deciembre 1949. Med. Clín., 1950 (en prensa).

9. André-Thomas y de Ajuriaguerra, J. - Étude Sémiologique du Tonus Musculaire. Editions Médicales Flamımarion, Paris, 1949.

10. Monrad-Krohn, G. H. - Clinical examination of the nervous system. Correspondence. Arch. Neurol. a. Psychiat., 62:663-664, 1949.

11. Monrad-Krohn, G. H. - Clinical Examination of the Nervous System. Ed. 9. Lewis, London, 1948, pp. 180 y sig.

12. Dejerine, J. - Sémiologie des Affections du Système Nerveux. Ed. 2. Masson et Cie., Paris, 1914, pp. 943.

13. Sicard, J. A. y Cantaloube, P. - Les réflexes musculaires du pied et de la Inain. Presse Méd., 24:145, 1916.

14. Barraquer-Bordas, L. - De la nature clinique des réflexes dits "profonds" et de ce que l'on apelle leur "zone réflexogène". Monatsschr. f. Psychiat. u. Neurol., 120:31-37, 1950 .

15. Torruella-Font, J. - Comunicación personal al autor.

16. a) Barraquer-Ferré, L., de Gispert-Cruz, I. y Castañer-Vendrell, E. - Tratado de Enfermedades Nerviosas. Salvat Ed., Barcelona, 1936-1940. b) BarraquerFerré, L. - Diagnóstico clínico precoz de la sífilis nerviosa. Medicamenta, 2:201-206, 1944. c) Barraquer-Bordas, L. - Estudios sobre fisiopatologia neuromuscular. II: El reflejo del tríceps sural, la contracción idiomuscular y e] fenómeno del contraste. Clín. y Laborat., 47:326-246, 1949.

17. Babinski, J. - Oeuvre Scientifique. Masson et Cie., Paris, 1934, pp. 451 y sig.

Lauria 102-Barcelona, España. 